\title{
A Photodetector-Driven Coherent RF Array with Wide Tuning Range
}

\author{
Behrooz Abiri ${ }^{1}$, Craig Ives ${ }^{*}$, and Ali Hajimiri ${ }^{1}$ \\ ${ }^{I}$ Department of Electrical Engineering, California Institute of Technology, Pasadena, CA 91125, USA \\ *cives@caltech.edu
}

\begin{abstract}
A sixteen-element coherent array of wideband spiral antennas driven by photodetectors is presented. The array radiates between 21 and $65 \mathrm{GHz}$, with $-45 \mathrm{dBm}$ of coupled power at $42 \mathrm{GHz}$. (C) $2019 \mathrm{The}$ Author(s)
\end{abstract}

OCIS codes: (130.0130) Integrated optics; (130.3120) Integrated optics devices

\section{Introduction}

A low cost, simple, and reliable integrated source for generating coherent RF and millimeter waves could enable a new generation of sensors and communications technology, which is essential for enabling $5 \mathrm{G}$ networks and the internet of things (IoT) [1]. Previous work has approached the photonic generation of RF signals using multi-port driven radiators [2], uni-traveling carrier photodiodes [3], and photoconductive antennas [4], among other means. There has also been significant work on generating RF and mm-wave power with electronics, including distributed active radiators [5] and self-feeding oscillators [6]. In contrast to these works, the design presented here is lowcomplexity, making array scaling easier, and fabricated entirely in a silicon photonic process, ensuring compatibility with CMOS.

In this work, a photodetector-driven array of spiral antennas generates RF power within the frequency range of 21 to $65 \mathrm{GHz}$ using a simple architecture that is readily scalable. A photonics-based design circumvents many of the challenges associated with generating high frequency radiation electronically, which requires complex designs to account for the parasitics or $f_{\max }$ of a process. A photodetector-driven radiator instead accepts light from two coherent (laser) sources, which beat in the photodetector and produce a mixed component at the difference between the laser wavelengths. This mixed component, which is at RF frequencies in this design, is then radiated by the array of antennas.

\section{Spiral antennas}

The individual photodetector for the demonstrated array is shown in Fig. 1a and 1b. It is dual fed to increase its power handling capacity and efficiency [7]. The waveguides are $220 \mathrm{~nm}$ tall and 500nm wide, but widen to $600 \mathrm{~nm}$ in the light absorbing region, which is $30 \mathrm{um}$ long. In this region, the germanium is 500nm wide and sits on top of the waveguide, giving a relatively small cross section. Because of the smaller cross section, the germanium absorbs less light per unit length than a standard photodetector in this process, again increasing its power handling capacity. The bandwidth of the photodiode in this particular structure is transit time limited, since only the fringes of the electric field can sweep carriers away.

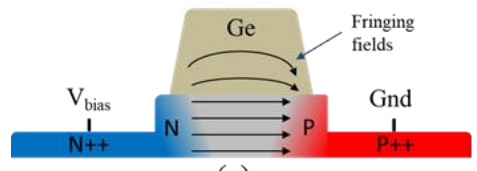

(a)

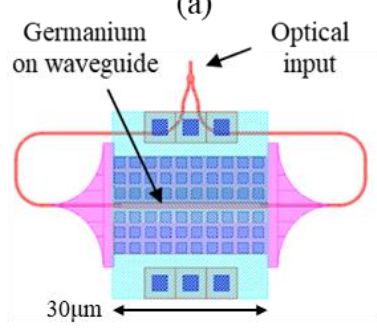

(b)

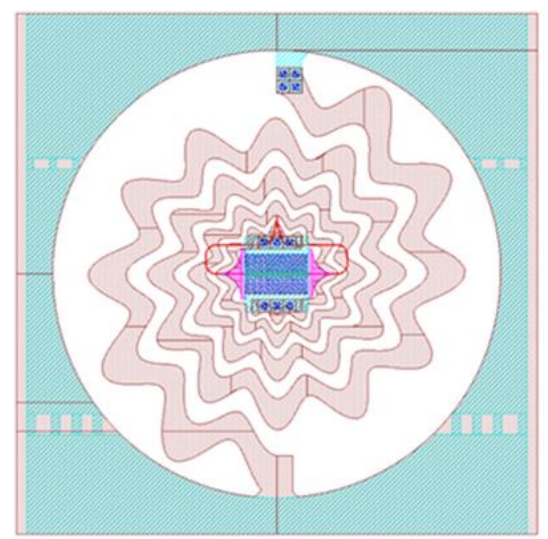

(c)

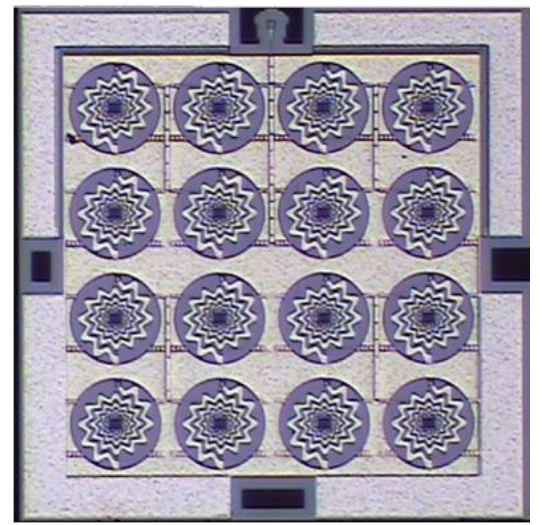

(d)

Figure 1: a) Cross section of the photodiode. b) Layout of the individual germanium photodiode. c) Layout of a radiating element. d) Micrograph of the chip, which has a die size of $1.2 \times 1.2 \mathrm{~mm}$. 
The photodetector drives a wideband log-periodic spiral antenna with sinusoidal corrugation on the spiral, which increases the effective length of the spiral to reduce the antenna's lower cutoff frequency, without increasing the physical size of the antenna. The DC bias of the photodetector is provided by the spiral antenna arms. This design eliminates the need for a separate balun and RF choke and integrates those functionalities with the antenna and the photodetector. The spiral antenna's impedance is inductive at its radiating frequencies, which helps cancel out the photodiode capacitance.

\section{Coherent array}

The coherent array is formed by matching the optical path lengths for each radiating element with an H-tree distribution structure, resulting in an element spacing of $250 \mu \mathrm{m}$. Each spiral has a nominal upper cutoff above $3 \mathrm{THz}$; however, the photodiode response falls off far below the spiral antenna half-wavelength cutoff (Fig. 2b), resulting in a reduction of the radiation efficiency within the photodiode's bandwidth.

\section{Measurement results}

Coupled power from the spiral array is shown in Fig. 2a, measured between 21 and $65 \mathrm{GHz}$, with $30.2 \mathrm{dBm}$ input optical power. The array radiates $-45 \mathrm{dBm}$ at $42.4 \mathrm{GHz}$. It can be observed that the radiation efficiency of the spiral antennas is limiting the radiated power at lower frequencies, while the bandwidth of the photodiode is limiting the power at higher frequencies. In the measurement setup of Fig. 2c, the chip is placed on a glass slide which is then placed on top of the horn aperture. When there is an optical input, the radiation is detected from the backside of the chip.

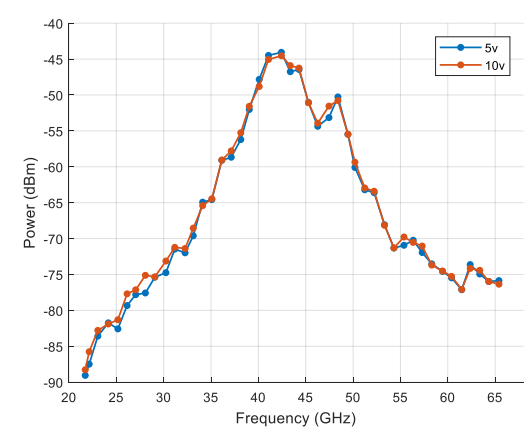

(a)

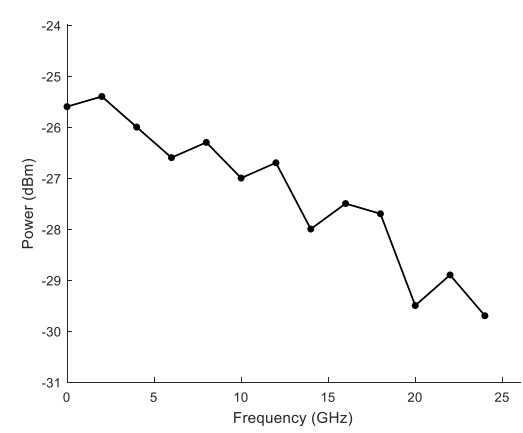

(b)

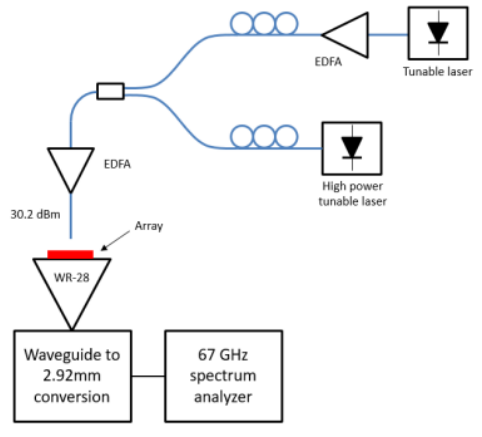

(c)

Figure 2: a) Coupled power from the array when reverse biased at $5 \mathrm{~V}$ and $10 \mathrm{~V}$. Each data point is the average of ten measurements, with steps of $1 \mathrm{GHz}$. b) Frequency response of a single photodiode. c) The experimental setup for measuring radiated power. For the 40-67 GHz band, the WR-28 horn is replaced with a WR-15 horn.

\section{References}

[1] J. G. Andrews, S. Buzzi, W. Choi, S. V. Hanly, A. Lozano, A. C. K. Soong and J. C. Zhang, "What Will 5G Be?," IEEE Journal on Selected Areas in Communications, vol. 32, no. 6, pp. 1065-1082, 2014.

[2] S. M. Bowers, B. Abiri, F. Aflatouni and A. Hajimiri, "A Compact Optically Driven Travelling-Wave Radiating Source," in Optical Fiber Communication Conference, San Francisco, 2014.

[3] K. Li, X. Xie, Q. Li, Y. Shen, M. E. Woodsen, Z. Yang, A. Beling and J. C. Campbell, "High-Power Photodiode Integrated With Coplanar Patch Antenna for 60-GHz Applications," IEEE Photonics Technology Letters, vol. 27, no. 6, pp. 650-653, 2015.

[4] C. W. Berry, M. R. Hashemi and M. Jarrahi, "Generation of high power pulsed terahertz radiation using a plasmonic photoconductive emitter array with logarithmic spiral antennas," Appl. Phys. Lett., vol. 104, no. 8, 2014.

[5] K. Sengupta and A. Hajimiri, "A $0.28 \mathrm{THz}$ power-generation and beam-steering array in CMOS based distributed active radiators," IEEE Journal of Solid-State Circuits, vol. 47, no. 12, pp. 3013-3031, 2012.

[6] Z. Hu and R. Han, "Fully-scalable 2D THz radiating array: A 42-element source in 130-nm SiGe with 80-uW total radiated power at 1.01 THz," in IEEE Radio Frequency Integrated Circuits Symposium, Honolulu, 2017.

[7] C.-M. Chang, J. H. Sinsky, P. Dong, G. de Valicourt and Y.-K. Chen, "High-power dual-fed traveling wave photodetector circuits in silicon photonics," Optics Express, vol. 23, no. 17, 2015. 